\title{
Intradialytic cycling does not exacerbate microparticles or circulating markers of systemic inflammation in haemodialysis patients
}

\author{
Patrick J. Highton ${ }^{1,8} \cdot$ Daniel S. March ${ }^{2,3} \cdot$ Darren R. Churchward ${ }^{2} \cdot$ Charlotte E. Grantham ${ }^{2} \cdot$ Hannah M. L. Young ${ }^{3,4}$. \\ Matthew P. M. Graham-Brown ${ }^{2,3}$. Seila Estruel ${ }^{6} \cdot$ Naomi Martin $^{7} \cdot$ Nigel J. Brunskill $^{2,3}$ • Alice C. Smith ${ }^{3,5}$. \\ James O. Burton ${ }^{1,2,3} \cdot$ Nicolette C. Bishop $^{1}$ (])
}

Received: 8 September 2021 / Accepted: 9 November 2021 / Published online: 2 December 2021

(c) The Author(s) 2021

\begin{abstract}
Purpose Patients receiving haemodialysis (HD) display elevated circulating microparticle (MP) concentration, tissue factor (TF) expression and markers of systemic inflammation, though regular intradialytic cycling (IDC) may have a therapeutic effect. This study investigated the impact of regular, moderate-intensity IDC on circulating MPs and inflammatory markers in unit-based HD patients.

Methods Patients were cluster-randomised to intervention $(n=20$, age: $51.4 \pm 18.1$ years, body mass: $77.6 \pm 18.3 \mathrm{~kg}$, mean \pm SD) or no-exercise control $(n=20,56.8 \pm 14.0$ years, $80.5 \pm 26.5 \mathrm{~kg})$. Intervention participants completed 30 min of moderate intensity (rating of perceived exertion [RPE] of 12-14) IDC, thrice weekly for 6 months. Pre-dialysis venous blood samples were obtained at 0, 3 and 6 months. Circulating MP phenotypes, cytokines, chemokine and MP TF expression were quantified using flow cytometry and cytometric bead array assays.

Results Despite high exercise compliance (82\%), no IDC-dependent effects were observed for any MP, cytokine or chemokine measure $\left(p \geq 0.051, \eta_{\rho}^{2} \leq 0.399\right)$ other than TNF- $\alpha\left(p=0.001, \eta_{\rho}^{2}=0.186\right)$, though no significance was revealed upon post hoc analysis.

Conclusion Six months of regular, moderate-intensity IDC had no effect on MPs, cytokines or chemokines. This suggests that the exercise did not exacerbate thrombotic or inflammatory status, though further functional assays are required to confirm this.
\end{abstract}

Trial registration ISRCTN1129707, prospectively registered on 05/03/2015.

Keywords Aerobic exercise $\cdot$ End-stage renal disease $\cdot$ Haemodialysis $\cdot$ Microparticles $\cdot$ Intradialytic cycling · Inflammation

Abbreviations

ANOVA Analysis of variance

$\mathrm{CD} \quad$ Cluster of differentiation

Communicated by Fabio Fischetti.

Nicolette C. Bishop

N.C.Bishop@lboro.ac.uk

1 School of Sport, Exercise and Health Sciences, Loughborough University, Loughborough, UK

2 Department of Cardiovascular Sciences, University of Leicester, Leicester, UK

3 John Walls Renal Unit, Leicester General Hospital, University Hospitals of Leicester, Leicester, UK

4 Department of Respiratory Sciences, University of Leicester, Leicester, UK

$\begin{array}{ll}\text { CV } & \text { Coefficient of variation } \\ \text { ESKD } & \text { End-stage kidney disease } \\ \text { HD } & \text { Haemodialysis } \\ \text { IDC } & \text { Intradialytic cycling } \\ \text { IL } & \text { Interleukin }\end{array}$

5 Department of Health Sciences, University of Leicester, Leicester, UK

6 Department of Physiological Sciences, University of Barcelona, Barcelona, Spain

7 Leicester School of Allied Health Sciences, Faculty of Health and Life Sciences, De Montfort University, Leicester, UK

8 Present Address: NIHR Applied Research Collaboration East Midlands, Leicester Diabetes Centre of Research, University of Leicester, Leicester, UK 


$\begin{array}{ll}\text { MCP } & \text { Monocyte chemotactic protein } \\ \text { MP } & \text { Microparticle } \\ \text { RPE } & \text { Rate of perceived exertion } \\ \text { SD } & \text { Standard deviation } \\ \text { SPSS } & \text { Statistical package for the social sciences } \\ \text { TF } & \text { Tissue factor } \\ \text { TNF- } \alpha & \text { Tumour necrosis factor-alpha }\end{array}$

\section{Introduction}

People with end-stage kidney disease (ESKD) receiving regular haemodialysis (HD) display elevated cardiovascular mortality, and cardiovascular disease is the leading cause of death in this population (23.3\%) (UK Renal Registry 2019). This may be in part due to elevated circulating microparticle (MP) concentrations and altered MP characteristics. MPs are shed upon cellular activation or apoptosis and can act as biomarkers for inflammation and leukocyte dysfunction whilst also promoting thrombosis through tissue factor (TF) expression (Piccin et al., 2007). HD treatment causes increased platelet and neutrophil activation and thus increases MP shedding (Daniel et al. 2006). Furthermore, MPs isolated from HD patients display elevated thrombotic potential compared to the healthy population (Burton et al. 2013) and may be predictive of mortality (Amabile et al. 2011).

HD patients also display chronically elevated circulating concentrations of pro-inflammatory cytokines (e.g. interleukin-6 (IL-6), tumour necrosis factor-alpha (TNF- $\alpha$ )) (Rysz et al. 2006) and reduced circulating concentrations of antiinflammatory cytokines (e.g. IL-10) (Zhang et al. 2010). Potential causes include chronic uraemia-induced leukocyte ligation, altered leukocyte subset distributions or HD treatment-induced endotoxin exposure (Zhang et al. 2010); (Hauser et al. 2008; Heine et al. 2012). Chronic leukocyte activation also increases the chemokine secretion (e.g. monocyte chemotactic protein-1 (MCP-1)), which positively associates with systemic inflammation (Papayianni et al. 2002) and may drive atherosclerosis in HD patients (Hu et al. 2016).

Both acute aerobic exercise and regular aerobic exercise training can promote favourable changes in MPs (i.e. reduced total and TF + MP concentration) (Babbitt et al. 2013; Highton et al. 2019), an effect which has also been observed in chronic kidney disease and renal transplantation (Highton et al. 2020). In addition, regular aerobic exercise training can reduce circulating pro-inflammatory cytokine and chemokine concentrations (Gleeson et al. 2011; Trøseid et al. 2004), and therefore, may be therapeutic for HD patients. However, HD patients report lack of time and opportunity, as well as safety fears as barriers to traditional exercise participation (Delgado and Johansen 2011). Intradialytic cycling (IDC), which is completed during routine
HD treatment under the supervision of exercise professionals or nursing staff, circumnavigates these issues and has been shown to reduce arterial stiffness and cardiovascular risk (Toussaint et al. 2008).

Only one study has investigated the impact of IDC on MPs (Martin et al. 2018), reporting that an acute bout of IDC induced an increase in MP reactive oxygen species release but no change in MP numbers. This is theorised to promote a long-term anti-inflammatory environment through repeated exposure and adaptation, though the long-term effects have yet to be investigated.

An acute bout of moderate-intensity IDC may also prevent an HD-induced increase in IL-6 (Wong et al. 2017) (though non-significantly) or increase circulating IL-10 concentrations to a greater degree than that seen in nonexercising patients (Peres et al. 2015), though some studies investigating regular IDC training have found no impact on inflammatory markers (Dungey et al. 2017; Gołębiowski et al. 2012; Toussaint et al. 2008). However, previous research has exhibited methodological limitations, such as taking resting samples after HD initiation (Dungey et al. 2017), including inappropriate control groups (Gołębiowski et al. 2012) or using small sample sizes (Toussaint et al. 2008). Therefore, there is need for research investigating the impact of regular IDC on MPs and systemic inflammation that addresses these methodological issues. The aim of this study was to investigate how regular, moderate-intensity IDC affects resting MP concentration, TF expression and cytokine and chemokine concentrations.

\section{Materials and methods}

\section{Ethics and study design}

Participants were recruited as a subsample of the 'CYCLEHD' study (ISRCTN1 129707), the protocol (Graham-Brown et al. 2016) and primary outcome findings (Graham-Brown et al. 2021) of which have been published previously. Favourable opinion was provided by the NHS Research Ethical Committee (14/EM/1190). Participants were clusterrandomised based on their regular HD shift cohort to usualcare control or intervention groups. Outcome assessments were completed at baseline, 3 and 6 months.

\section{The intervention}

Participants first completed a 1-month familiarisation period, during which they familiarised themselves with the exercise bike and gradually built up their exercise duration to achieve the desired $30 \mathrm{~min}$ by the end of this period. Participants then completed 6 months of structured, thrice-weekly moderate-intensity IDC using a cycle ergometer (Letto 2, 
Motomed, Reck, Germany). Each bout consisted of $30 \mathrm{~min}$ of cycling plus 5 min warm-up and cool-down, at an average cadence of 60-70 rpm and a rating of perceived exertion of 12-14. Cycling resistance was progressively increased to maintain RPE in response to exercise adaptation (GrahamBrown et al. 2016). Relevant clinical data, including routine laboratory results, were extracted from medical records.

\section{Blood sampling}

Resting blood samples were taken prior to HD initiation on the second or third HD shift of the week (i.e. after the short inter-dialytic break). $30 \mathrm{~mL}$ of venous blood was drawn directly into monovettes containing either $\mathrm{K}_{3}$ EDTA or sodium citrate (Sarstedt, Nümbrecht, Germany). Sodium citrate-treated blood was centrifuged at $2500 \mathrm{~g}$ for $15 \mathrm{~min}$ at $20{ }^{\circ} \mathrm{C}$, after which the top $90 \%$ was removed and centrifuged again at $2500 \mathrm{~g}$ for $15 \mathrm{~min}$ at $20^{\circ} \mathrm{C}$ - the supernatant (platelet-free plasma) was then removed and stored in $250 \mu \mathrm{l}$ aliquots at $-80{ }^{\circ} \mathrm{C}$ for future MP analysis. $\mathrm{K}_{3}$ EDTA-treated blood was centrifuged at $2500 \mathrm{~g}$ for $10 \mathrm{~min}$ at $4{ }^{\circ} \mathrm{C}$ and the supernatant was separated and stored in $500 \mu \mathrm{l}$ aliquots at $-80{ }^{\circ} \mathrm{C}$ for future cytokine and chemokine analysis.

\section{Laboratory analysis}

MPs were categorised as platelet-derived $\left(\mathrm{CD} 42 \mathrm{~b}^{+}\right)$, neutrophil-derived $\left(\mathrm{CD} 6 \mathrm{~b}^{+}\right)$, monocyte-derived $\left(\mathrm{CD} 14^{+}\right)$and endothelial cell-derived $\left(\mathrm{CD} 144^{+}\right)$, whilst prothrombotic potential was assessed using TF $\left(\mathrm{CD} 142^{+}\right)$expression. Aliquots of platelet-free plasma were thawed at room temperature prior to analysis. Samples and antibodies were combined and incubated in the dark for $25 \mathrm{~min}$ at controlled room temperature $\left(20^{\circ} \mathrm{C}\right)$. The isotype controls used were anti-IgG1k PE, anti-IgG1k APC, anti-IgMk PE and antiIgG2ak PE. The antibodies used for assessing MPs were anti-AnV FITC, anti-CD42b PE, anti-CD66b PE, anti-CD14 $\mathrm{PE}$, anti-CD144 PE and anti-CD142 APC. Following incubation, samples were analysed using an Accuri C6 flow cytometer for $2 \mathrm{~min}$ at a 'slow' sampling rate $\left(14 \mu \mathrm{L} \cdot \mathrm{min}^{-1}\right)$ and events were collected into previously established plots (Fig. 1) (CV 13.88\%). This methodology and gating strategy was adapted from previous research from our group (Martin et al. 2018) and has been used previously in the general population (Highton et al. 2019) and in those with chronic kidney disease (Highton et al. 2020).

Plasma concentrations of circulating cytokines (IL-2, IL-6, IL-10, IL-17a, and TNF- $\alpha$ ) and chemokines (IL-8, MCP-1) were analysed using a cytometric bead array and BD Accuri C6 flow cytometer (BD Biosciences, Oxford, UK). Chemokines were analysed using a pre-configured kit (552990), whilst cytokines were analysed using an enhanced-sensitivity flex-set, combining a master buffer kit
(561523) with the corresponding cytokine kits (CV 10.08\%). Samples that fell below the minimum detectable threshold $(0.274 \mathrm{pg} / \mathrm{mL})$ were assigned a value of $0.01 \mathrm{pg} / \mathrm{mL}$ and included in the analyses.

\section{Statistical analysis}

Non-normally distributed data were logarithmically transformed to allow for parametric testing, though all data are presented as non-transformed. Baseline group differences were compared using independent samples t-tests. Exercise progression data were analysed using one-way ANOVAs. The impact of the exercise intervention was assessed using a mixed design ANOVAs. Effect sizes were estimated using partial eta squared $\left(\eta_{\rho}^{2}, 0.02=\right.$ small, $0.13=$ medium, $0.26=$ large) (Bakeman 2005). Upon detection of an interaction effect, independent samples t-tests were completed at each time-point to elucidate the effect. $p<0.05$ was considered statistically significant. Data are presented as 'mean \pm SD'. Statistical analysis was completed on IBM SPSS version 25.0 (Chicago, Illinois) and graphs were produced on GraphPad Prism (v.7 GraphPad Software Inc., CA, USA).

\section{Results}

\section{Participants}

Baseline demographic and clinical data are presented in Table 1.

\section{Exercise sessions}

In total, $84 \%$ of all IDC sessions were completed ( $\pm 13 \%$, range 48-99\%). The mean gear (indicative of intensity) achieved during each session increased from baseline to months five $(p=0.026)$ and six $(p=0.024)$. No other exercise session characteristics changed throughout the programme (data not shown).

\section{Circulating microparticles}

MP results are presented in Fig. 2 and Table 2. Both total MPs and PS/Annexin- $\mathrm{V}^{+}$MPs were similar at baseline with no evidence of IDC-dependent effects $(p \geq 0.326$, $\eta_{\rho}^{2} \leq 0.009$ ). There was also no evidence for IDC-dependent effects observed in the circulating concentration of total platelet-derived MPs or $\mathrm{TF}^{+}$platelet-derived MPs, or the \% of total MPs that were platelet-derived or platelet-derived MPs that were $\mathrm{TF}^{+}\left(p \geq 0.279, \eta_{\rho}^{2} \leq 0.035\right)$.

The number of neutrophil-derived MPs was similar in both groups at baseline $(p=0.279)$. Although there was an 

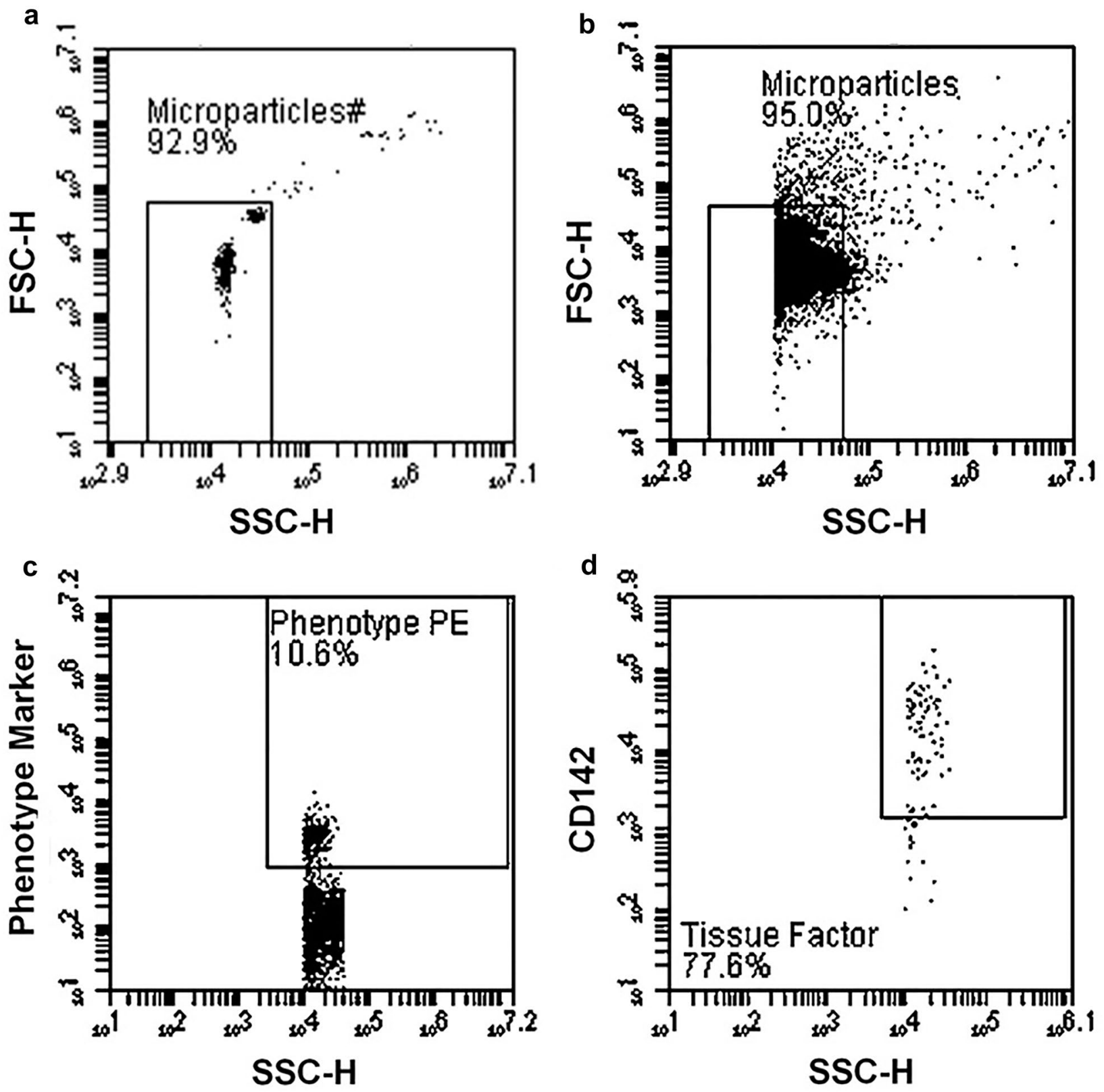

Fig. 1 MP flow cytometry gating strategy. A Mega-mix beads (BioCytex, Theale, UK, product reference 7801) of known size $(0.5 \mu \mathrm{m}$, $0.9 \mu \mathrm{m}$, and $3.0 \mu \mathrm{m}$ ) to determine subsequent size gating. Two distinct populations ( 0.5 and $0.9 \mu \mathrm{m}$ beads, shown in red) are visible, and thus the gate can be set to include these populations whilst excluding the $3.0 \mu \mathrm{m}$ population (not visible here). B 'All MPs' based on size, set using previous mega-mix beads. The minimum trigger threshold of $0.3 \mu \mathrm{m}$ is necessary to exclude the noise floor inher-

increase in the number of neutrophil-derived MPs over time in the control group, there was insufficient evidence overall (time* group interaction effect $p=0.051, \eta_{\rho}^{2}=0.096$ ) to demonstrate a difference between exercise and control groups after 6 months $(p=0.063)$ (Fig. 2a). In addition, no other neutrophil-derived MP variable $\left(\mathrm{TF}^{+}\right.$neutrophil-derived ent in all cytometers, hence why the visible MP population (known to be comprised of particles ranging from 0.1 to $1.0 \mu \mathrm{m}$ in diameter) is cut off at around $10^{4}$ on the $X$ axis. C Phenotype marker expression (CD14, CD42b, CD66b, CD144-all four phenotypes employed the same gating strategy), used to quantify MPs of different cellular sources. D Tissue Factor expression, back-gated onto the previous graph $(\mathbf{C})$ to identify those particles presenting as positive for both their phenotype marker and TF (CD142)

MPs, $\%$ of total MPs that were neutrophil-derived MPs, \% of neutrophil-derived MPs that were $\mathrm{TF}^{+}$) displayed evidence of time* group interaction effects $\left(p \geq 0.261, \eta_{\rho}{ }^{2} \leq 0.040\right)$.

Similarly, the circulating concentration of total and $\mathrm{TF}^{+}$ monocyte or endothelial-derived MPs, the \% of total MPs that were monocyte or endothelial-derived and the $\%$ of 
Table 1 Baseline demographic data

\begin{tabular}{lll}
\hline Group & Control $(n=20)$ & Exercise $(n=20)$ \\
\hline Age (years) & $56.8 \pm 14.0$ & $51.4 \pm 18.1$ \\
Male gender, $n$ & 12 & 12 \\
Height $(\mathrm{m})$ & $1.67 \pm 0.08$ & $1.66 \pm 0.11$ \\
Body mass $(\mathrm{kg})$ & $80.49 \pm 26.47$ & $77.61 \pm 18.27$ \\
BMI $\left(\mathrm{kg} / \mathrm{m}^{2}\right)$ & $28.67 \pm 7.53$ & $28.12 \pm 6.44$ \\
Systolic BP $(\mathrm{mmHg})$ & $145 \pm 19$ & $142 \pm 20$ \\
Diastolic BP $(\mathrm{mmHg})$ & $72 \pm 11$ & $74 \pm 13$ \\
Dialysis vintage $(\mathrm{months})$ & $21.6 \pm 19.3$ & $33.0 \pm 27.1$ \\
Hypertension $(n, \%)$ & $16(80)$ & $15(75)$ \\
Diabetes $(n, \%)$ & $9(45)$ & $5(25)$ \\
\hline
\end{tabular}

Where appropriate, data are presented as 'mean $\pm \mathrm{SD}$ '

monocyte or endothelial-derived MPs that were $\mathrm{TF}^{+}$all showed no evidence of any baseline differences $(p \geq 0.343)$ or time*group interaction effects $\left(p \geq 0.097, \eta_{\rho}{ }^{2} \leq 0.399\right)$ (Fig. 2b, c).

\section{Circulating cytokines and chemokines}

All IL-2 values fell below the minimum detectable threshold and so analysis was not completed. All samples $(n=40)$ provided detectable IL-6 and IL-10 values (Fig. 3a, b), though there was insufficient evidence to suggest the presence of baseline differences or interaction effects $(p \geq 0.060$, $\left.\eta_{\rho}^{2} \leq 0.076\right)$.

Two control and seven IDC participants displayed IL17 a results above the minimum detectable threshold - the remainder were assigned as $0.01 \mathrm{pg} / \mathrm{mL}$. No baseline group differences or interaction effects were observed ( $p \geq 0.262$, $\left.\eta_{\rho}^{2} \leq 0.150\right)$

TNF- $\alpha$ values above the minimum detectable threshold were observed in 9 control participants and 12 IDC participants, whilst the remaining values were assigned as $0.01 \mathrm{pg} / \mathrm{mL}$. No baseline group differences were observed $(p=0.294)$, though there was strong evidence to suggest a significant time* group interaction effect $(p=0.001$, $\left.\eta_{\rho}{ }^{2}=0.186\right)$. However, no further statistical significance was detected upon post hoc analysis (Fig. 3c).

Circulating concentrations of IL- 8 and MCP-1 displayed no evidence of baseline differences $(p \geq 0.101)$ or time* group interaction effects $\left(p \geq 0.151, \eta_{\rho}{ }^{2} \leq 0.053\right)$ (Table 3).

\section{Clinical laboratory outcomes}

No evidence was observed to suggest that the intervention had any effects on relevant clinical results obtained from medical records (Table 4).

\section{Discussion}

This study found no evidence to suggest that a 6-month programme of thrice-weekly structured IDC training exacerbates circulating MP, cytokine, or chemokine concentrations or MP tissue factor expression.

Whilst a reduction in neutrophil-derived MP concentration was observed in the IDC group, thus suggesting a potential anti-inflammatory effect of regular IDC, there was insufficient evidence $(p=0.051)$ to firmly support this result, and therefore, this finding should be considered hypothesis-generating only. If future research reveals stronger evidence for this effect, possible mechanistic explanations that could be investigated include reduced uraemia-induced neutrophil activation or reduced neutrophil apoptosis resulting in reduced neutrophil-derived MP shedding. However, whilst the HD procedure can drive leukocyte apoptosis (Moser et al. 2003), the impact of IDC has not yet been investigated. As HD patients commonly experience impaired circulation (McIntyre et al. 2008), regular IDC training may restore systemic circulation and prevent hypoxia, reducing neutrophil activation. A reduction in circulating neutrophil-derived MPs would be expected to represent an anti-inflammatory effect, which could ultimately reduce thrombosis and cardiovascular risk, however, the impact of IDC in this regard can only be postulated based on the findings from this study.

Circulating concentrations of MPs derived from endothelial cells, platelets and monocytes are increased in patients with renal failure, and associate with vascular dysfunction (Daniel et al. 2008). The MP results of this study mimic those investigating acute IDC (Martin et al. 2018), and as such it may be that the exercise was not of a sufficient intensity in either case to elicit significant reductions in circulating MP concentrations, either acutely or on a long-term basis. However, as the mean power outputs achieved in this study were similar to those of previous IDC research ( 21-22 W) (Dungey et al. 2015; Martin et al. 2018), this exercise intensity is likely to be representative of what can be reasonably expected of HD patients. As such, whilst regular aerobic exercise has been previously reported to elicit MP-mediated health benefits in other populations (Highton et al. 2018), there is as yet insufficient evidence to suggest that this can be achieved with regular IDC.

Supporting this, whilst previous research investigating acute IDC has suggested the possibility of an MPmediated anti-inflammatory effect of long-term IDC (Martin et al. 2018), this was not clearly observed here. Therefore, it may be that any possible transient effect of acute moderate-intensity IDC on MPs is not sufficient to induce a negative feedback loop potent enough to elicit an 



- Control Patients

- Exercise Patients

- Control Patients

- Exercise Patients

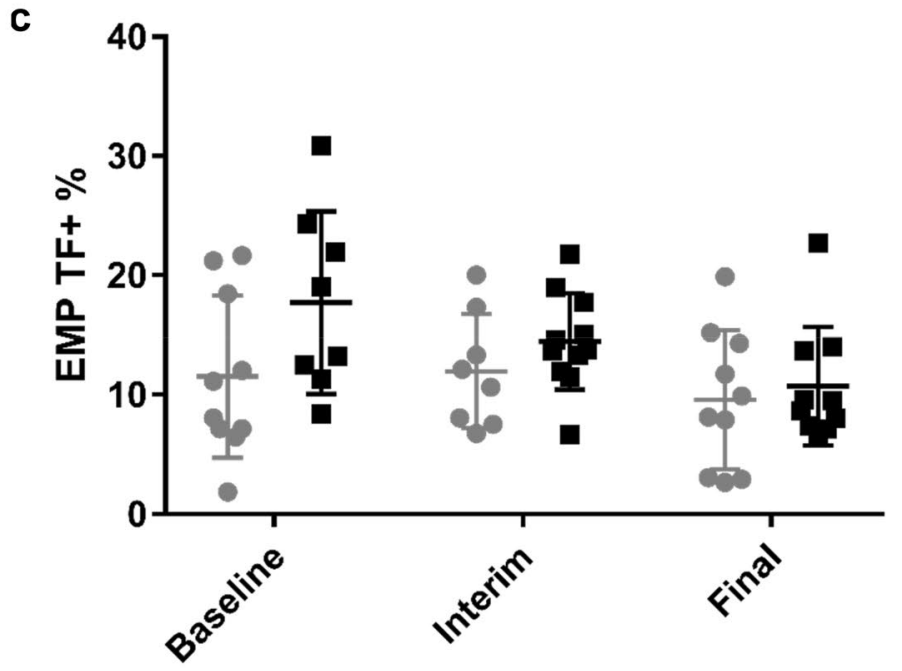

- Control Patients

- Exercise Patients 
4Fig. 2 Circulating concentration of neutrophil-derived MPs (NMP) (a), endothelial cell-derived MPs (EMP) (b) and the percentage of EMPs that express TF (c). Data are presented as 'mean \pm SD'. Concentration data were non-normally distributed and thus logarithmically transformed prior to analysis, whilst percentage data were normally distributed (control $n=20$, exercise $n=20$ )

overall anti-inflammatory effect. In addition, whilst previous research has displayed beneficial effects of regular moderate-intensity aerobic exercise on MP concentrations in the general population (Babbitt et al. 2013), this also was not observed here in this population of HD patients. This may be due in part to discrepancies in exercise intensity-Babbitt et al. incorporated objectively measured exercise intensity training $\left(65 \%\right.$ of $\left.\mathrm{VO}_{2 \max }\right)$ whilst here it was subjective (RPE 12-14), which may have resulted in a, respectively, lower achieved intensity. It may also be that the potent effect of end-stage renal failure and the HD procedure on MPs (Amabile et al. 2011; Daniel et al. 2006) prevents the observation of any effects induced by exercise of this intensity. However, there is currently no evidence to suggest that regular IDC exacerbates MP status in HD patients, though further functional assays would be required to investigate this more directly, for instance a prothrombinase assay to assess prothrombotic potential.

As there was insufficient evidence here to suggest the presence of ad MP-mediated anti-inflammatory response, it is unsurprising that no IDC-dependent effects were observed in circulating cytokines. The diverse battery of cytokines assessed here typically exhibit either pro-inflammatory effects (TNF- $\alpha$, IL-17a), anti-inflammatory effects (IL-2, IL-10), or both (IL-6) (Berg and Scherer 2005; Cortvrindt et al. 2017; Hoyer et al. 2008; Stenvinkel et al. 2005), though there was no evidence to suggest any exercise-dependent effects, either pro- or anti-inflammatory, despite initial evidence suggesting a reduction in TNF- $\alpha$. In previous research in the general population, both acute and regular aerobic exercise participation have elicited favourable changes in these cytokines (Gleeson et al. 2011; Rhind et al. 1996), and similar anti-inflammatory effects have also been displayed following acute IDC (Peres et al. 2015). A previous study investigating regular IDC also found no observable change in circulating cytokines despite observing reductions in circulating intermediate monocyte proportion, though at a slightly lower exercise intensity (16 W) (Dungey et al. 2017). It may be that HD patients cannot routinely achieve an exercise intensity sufficient enough to impact their resting systemic inflammatory profile. MCP-1 and IL-8, proinflammatory chemokines that promote leukocyte migration and associate with atherosclerosis and cardiovascular risk (Hashmi and Zeng 2006; Kusano et al. 2004; Papayianni et al. 2002), also displayed no IDC-dependent effects, likely for the same reason. However, it is encouraging that these circulating mediators were not exacerbated by regular IDC, which points towards the 'immunological safety' of this form of exercise in this population.

Table 2 MP phenotype and TF expression data

\begin{tabular}{|c|c|c|c|c|c|c|}
\hline & \multicolumn{3}{|l|}{ Control $(n=20)$} & \multicolumn{3}{|c|}{ Exercise $(n=20)$} \\
\hline & Baseline & Interim & Final & Baseline & Interim & Final \\
\hline Total MP conc. $\left(\times 10^{9} / \mathrm{L}\right) \#$ & $12.30 \pm 10.96$ & $12.26 \pm 18.29$ & $17.96 \pm 23.57$ & $14.76 \pm 17.84$ & $22.29 \pm 29.01$ & $20.71 \pm 20.08$ \\
\hline $\mathrm{AnV}^{+} \mathrm{MP}$ conc. $\left(\times 10^{7} / \mathrm{L}\right) \#$ & $13.59 \pm 9.30$ & $9.08 \pm 7.02$ & $17.03 \pm 12.25$ & $11.15 \pm 7.20$ & $10.56 \pm 7.20$ & $12.72 \pm 6.98$ \\
\hline PMP conc. $\left(\times 10^{7} / \mathrm{L}\right) \#$ & $7.91 \pm 4.52$ & $6.33 \pm 7.56$ & $8.48 \pm 5.63$ & $9.19 \pm 3.98$ & $10.21 \pm 7.87$ & $8.87 \pm 6.08$ \\
\hline $\mathrm{TF}^{+}$PMP conc. $\left(\times 10^{7} / \mathrm{L}\right)$ & $2.26 \pm 1.43$ & $1.72 \pm 1.61$ & $1.98 \pm 1.57$ & $2.69 \pm 1.65$ & $2.52 \pm 1.30$ & $2.41 \pm 1.52$ \\
\hline$\%$ PMP of total MPs & $1.59 \pm 1.92$ & $0.85 \pm 1.03$ & $1.75 \pm 2.59$ & $1.99 \pm 2.73$ & $1.42 \pm 1.61$ & $1.84 \pm 2.70$ \\
\hline$\% \mathrm{PMP} \mathrm{TF}^{+}$ & $28.46 \pm 14.40$ & $29.60 \pm 15.52$ & $23.70 \pm 12.25$ & $29.83 \pm 13.91$ & $32.30 \pm 12.79$ & $31.08 \pm 11.98$ \\
\hline $\mathrm{TF}^{+} \mathrm{NMP}$ conc. $\left(\times 10^{7} / \mathrm{L}\right) \#$ & $0.13 \pm 0.17$ & $0.24 \pm 0.40$ & $0.20 \pm 0.31$ & $0.17 \pm 0.27$ & $0.11 \pm 0.18$ & $0.15 \pm 0.22$ \\
\hline$\%$ NMP of total MPs & $0.24 \pm 0.49$ & $0.08 \pm 0.13$ & $0.20 \pm 0.36$ & $0.21 \pm 0.54$ & $0.07 \pm 0.13$ & $0.11 \pm 0.27$ \\
\hline$\% \mathrm{NMP} \mathrm{TF}^{+}$ & $19.84 \pm 12.25$ & $23.57 \pm 11.05$ & $18.90 \pm 8.77$ & $23.78 \pm 10.69$ & $26.21 \pm 10.29$ & $23.60 \pm 5.99$ \\
\hline MMP conc. $\left(\times 10^{7} / \mathrm{L}\right) \#$ & $3.82 \pm 3.04$ & $3.78 \pm 3.53$ & $3.91 \pm 3.35$ & $5.03 \pm 3.53$ & $4.21 \pm 3.49$ & $4.00 \pm 3.94$ \\
\hline $\mathrm{TF}^{+} \mathrm{MMP}$ conc. $\left(\times 10^{7} / \mathrm{L}\right) \#$ & $0.84 \pm 0.76$ & $0.73 \pm 0.80$ & $0.86 \pm 0.89$ & $1.24 \pm 0.98$ & $0.85 \pm 0.67$ & $0.97 \pm 1.34$ \\
\hline$\%$ MMP of total MPs & $0.81 \pm 1.57$ & $0.51 \pm 0.80$ & $1.14 \pm 2.15$ & $0.97 \pm 1.48$ & $0.72 \pm 0.81$ & $1.09 \pm 1.92$ \\
\hline$\% \mathrm{MMP} \mathrm{TF}^{+}$ & $21.16 \pm 7.60$ & $18.92 \pm 6.75$ & $20.56 \pm 9.97$ & $24.14 \pm 10.00$ & $20.92 \pm 8.54$ & $23.90 \pm 6.39$ \\
\hline $\mathrm{TF}^{+}$EMP conc. $\left(\times 10^{7} / \mathrm{L}\right) \#$ & $0.06 \pm 0.13$ & $0.09 \pm 0.13$ & $0.05 \pm 0.09$ & $0.06 \pm 0.09$ & $0.10 \pm 0.13$ & $0.08 \pm 0.09$ \\
\hline$\%$ EMP of total MPs & $0.10 \pm 0.18$ & $0.16 \pm 0.31$ & $0.19 \pm 0.45$ & $0.08 \pm 0.18$ & $0.26 \pm 0.40$ & $0.32 \pm 0.63$ \\
\hline
\end{tabular}

All data are presented as 'mean \pm SD'

AnV Annexin-V; PMP platelet-derived MP; NMP neutrophil-derived MP; MMP monocyte-derived MP; EMP endothelial cell-derived MP; TF tissue factor

\#Indicates that data were non-normally distributed and thus were logarithmically transformed prior to analysis 




- Control Patients

- Exercise Patients

b

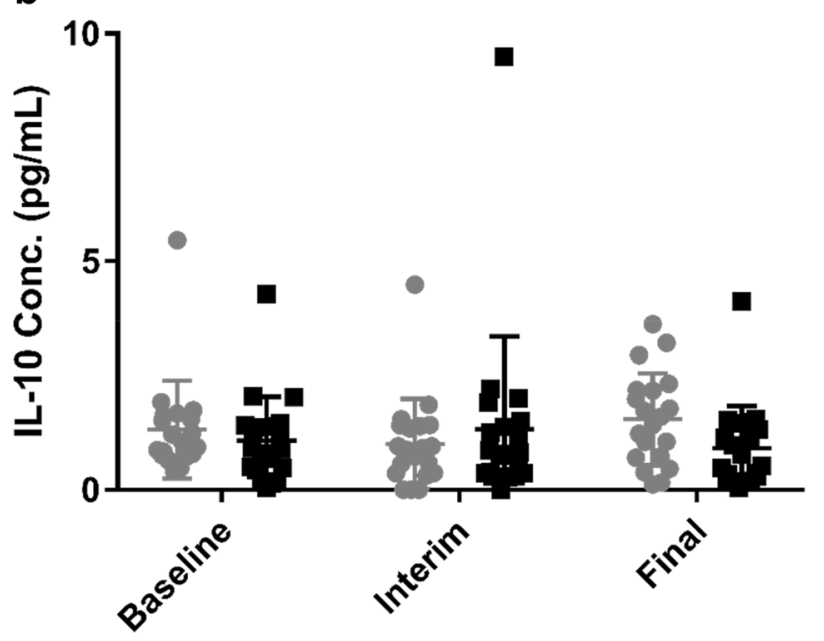

- Control Patients

- Exercise Patients

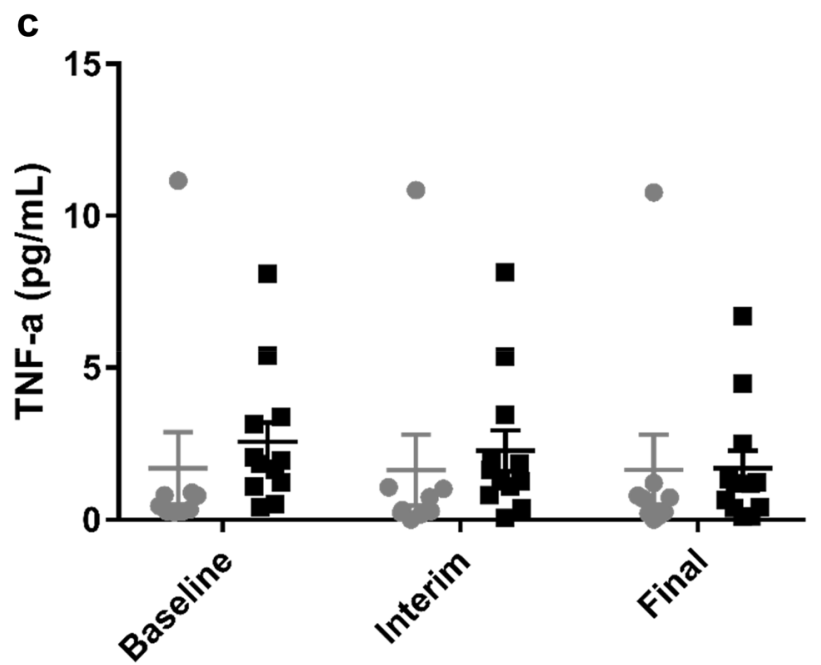

- Control Patients

- Exercise Patients

Fig. 3 Circulating IL-6 (a), IL-10 (b) and TNF- $\alpha$ (c) levels. Data are presented as 'mean \pm SD'. These data were non-normally distributed and thus logarithmically transformed prior to analysis (control $n=20$, IDC exercise $n=20$ ) 
Table 3 Circulating chemokine data

\begin{tabular}{|c|c|c|c|c|c|c|}
\hline \multirow[t]{2}{*}{ Chemokine } & \multicolumn{3}{|l|}{ Control $(n=20)$} & \multicolumn{3}{|c|}{ Exercise $(n=20)$} \\
\hline & Baseline & Interim & Final & Baseline & Interim & Final \\
\hline IL-8 (pg/mL) & $23.70 \pm 15.60$ & $20.09 \pm 13.90$ & $21.71 \pm 17.80$ & $20.68 \pm 9.61$ & $20.00 \pm 10.51$ & $21.41 \pm 11.67$ \\
\hline MCP-1 (pg/mL) & $47.06 \pm 23.47$ & $35.30 \pm 10.20$ & $44.64 \pm 18.25$ & $39.81 \pm 25.31$ & $39.65 \pm 22.94$ & $41.30 \pm 24.55$ \\
\hline
\end{tabular}

Data are presented as 'mean $\pm \mathrm{SD}$ '. These data were normally distributed

Table 4 Clinical laboratory results obtained from medical records at baseline, interim and final time-points

\begin{tabular}{lcllllll}
\hline & \multicolumn{3}{l}{ Control $(n=20)$} & & \multicolumn{5}{l}{ Exercise $(n=20)$} \\
\cline { 2 - 3 } & Baseline & Interim & Final & & Baseline & Interim & Final \\
\hline White cell count $\left(\times 10^{9} / \mathrm{L}\right)$ & $7.86 \pm 2.54$ & $7.03 \pm 1.97$ & $7.67 \pm 2.50$ & & $6.84 \pm 2.16$ & $6.64 \pm 1.57$ & $6.88 \pm 1.96$ \\
Neutrophils $\left(\times 10^{9} / \mathrm{L}\right)$ & $4.43 \pm 1.37$ & $4.58 \pm 1.18$ & $4.99 \pm 1.51$ & & $4.22 \pm 1.71$ & $4.39 \pm 1.48$ & $4.12 \pm 1.04$ \\
Lymphocytes $\left(\times 10^{9} / \mathrm{L}\right)$ & $1.8 \pm 0.5$ & $1.68 \pm 0.60$ & $1.61 \pm 0.60$ & & $1.7 \pm 0.8$ & $1.48 \pm 0.69$ & $1.73 \pm 0.61$ \\
Monocytes $\left(\times 10^{9} / \mathrm{L}\right)$ & $0.5 \pm 0.2$ & $0.54 \pm 0.09$ & $0.51 \pm 0.17$ & & $0.5 \pm 0.2$ & $0.48 \pm 0.15$ & $0.45 \pm 0.15$ \\
Total cholesterol $(\mathrm{mmol} / \mathrm{L})$ & $3.93 \pm 0.98$ & $4.08 \pm 1.08$ & $3.98 \pm 1.17$ & & $4.29 \pm 1.25$ & $4.29 \pm 1.70$ & $4.39 \pm 1.49$ \\
Triglycerides $(\mathrm{mmol} / \mathrm{L})$ & $1.85 \pm 1.50$ & $1.90 \pm 1.24$ & $1.64 \pm 0.98$ & & $1.65 \pm 1.02$ & $1.76 \pm 1.28$ & $1.74 \pm 0.71$ \\
Urea reduction ratio $(\%)$ & $74 \pm 14$ & $78 \pm 6$ & $76 \pm 6$ & & $77 \pm 8$ & $75 \pm 13$ & $75 \pm 9$ \\
\hline
\end{tabular}

No significant exercise-dependent effects were observed. All data were normally distributed and are presented as 'mean $\pm \mathrm{SD}$ '
Flow cytometry is considered the gold-standard technique most applicable to clinical research for the detection and characterisation of MPs due to its high throughput and the level of information it can provide concerning MP size, complexity and surface marker expression (Strasser et al. 2013; Van der Pol et al. 2014). In addition, flow cytometry allows for several steps of confirmation when detecting and measuring MPs, including confirmation based on size, internal complexity, and the expression of one or a combination of specific markers, which makes it easier to isolate the population of interest. Whilst this is possible with other techniques, such as nanoparticle-tracking analysis, it is more arduous and less comprehensive. Nanoparticle tracking analysis does have the advantage over traditional flow cytometry of being able to visualise the particles of interest (though this is possible with imaging flow cytometry (Lannigan and Erdbruegger 2017)). In addition, nanoparticle-tracking analysis typically provides a lower minimum detectable threshold $(0.05 \mu \mathrm{m})$ than this flow cytometry technique $(0.3 \mu \mathrm{m})$ (Highton et al. 2019), which is a previously identified limitation of flow cytometry (Burton et al. 2013). Therefore, an accepted limitation of the flow cytometry technique employed here is that it may be excluding a certain number of MPs below $0.3 \mu \mathrm{m}$ in diameter; however, it could also be argued that using nanoparticle-tracking analysis could also incorporate unwanted exosomes in the $0.05-0.1 \mu \mathrm{m}$ range in the analyses. Ideally, methodologies for isolating and analysing MP populations would employ two or more techniques to capitalise on the advantages of each technique and to ensure that the full size range is captured (Strasser et al. 2013; Szatanek et al. 2017). However, this was not possible in this study due to resource constraints, and therefore, flow cytometry was selected due to its advantages over other techniques (Szatanek et al. 2017). Further research should focus on harmonising the various techniques for MP detection to construct a comprehensive, standardised methodology.

\section{Limitations}

This study incorporated only a cohort of the full CYCLEHD study and as such was not informed by an a priori power calculation, though the sample size included was much larger than the majority of IDC studies that have investigated MPs or systemic inflammation in detail in the current body of literature. In addition, only one technique was employed for MP analysis which may have limited the range of captured particles, though this was due to the resource demand of employing more than one laboratory analysis technique. Lastly, the cytometric bead array technique was unable to detect certain cytokines (IL-2 and IL-17a) in a large number of samples suggesting insufficient assay sensitivity, though as IL- 6 and IL- 10 were detected above the minimum threshold in every sample, it is likely that the concentrations were simply too low. 


\section{Conclusions and future research}

In conclusion, this study found no evidence to suggest that a 6-month programme of thrice-weekly intradialytic cycling training altered circulating MP, cytokine or chemokine concentrations or MP tissue factor expression. Whilst this suggests the immunological safety of IDC, these findings can only be considered as hypothesis generating and should be pursued, primarily by incorporating further functional assays to investigate the impact on thrombotic potential and immune function. In addition, future research should aim to investigate the feasibility of methods to achieve greater exercise intensity using IDC, and whether this impacts MPs or the inflammatory environment in HD patients.

Acknowledgements The authors would like to thank Ruth Lead and Katie Logan for their contribution to the supervision of patient exercise sessions, the patients for their time and effort and the HD unit staff for their assistance in study delivery.

Author contributions JOB, NCB, ACS and NJB conceived and designed the research. PJH, DSM, DRC, CEG, HMLY, and MPMG-B supervised patient exercise and outcome assessment sessions. PJH and SE completed laboratory work, with supervision from NM and NCB. $\mathrm{PJH}$ analysed the data and wrote the manuscript. All the authors read and approved the manuscript.

Funding The CYCLE-HD study was funded by a grant from the National Institute of Health Research (CS-2013-13-014) and supported by the Stoneygate Trust. This research was also supported by the NIHR Leicester Biomedical Research Centre. Hannah Young is supported by a grant from the NIHR (DRF-2016-09-015). The views expressed in this publication are those of the authors and not necessarily those of the NHS, the National Institute for Health Research or the Department of Health. The funders had no role in the study design; collection, analysis, and interpretation of the data; writing the report, or the decision to submit the report for publication.

Data availability Data are available from the corresponding author upon reasonable request.

Code availability Not applicable.

\section{Declarations}

Conflict of interest The authors report no conflict of interest.

Ethics approval Favourable opinion was provided by the NHS Research Ethical Committee (14/EM/1190).

Consent to participate All the participants, after having the research study explained to them by a member of the research team, were given at least $48 \mathrm{~h}$ to consider their participation and ask any questions they might have had. Following this, willing participants provided written informed consent to a trained member of the study team.

Consent for publication Not applicable-no patient identifiable or personal information or images are presented in this manuscript.
Open Access This article is licensed under a Creative Commons Attribution 4.0 International License, which permits use, sharing, adaptation, distribution and reproduction in any medium or format, as long as you give appropriate credit to the original author(s) and the source, provide a link to the Creative Commons licence, and indicate if changes were made. The images or other third party material in this article are included in the article's Creative Commons licence, unless indicated otherwise in a credit line to the material. If material is not included in the article's Creative Commons licence and your intended use is not permitted by statutory regulation or exceeds the permitted use, you will need to obtain permission directly from the copyright holder. To view a copy of this licence, visit http://creativecommons.org/licenses/by/4.0/.

\section{References}

Amabile N, Guérin AP, Tedgui A, Boulanger CM, London GM (2011) Predictive value of circulating endothelial microparticles for cardiovascular mortality in end-stage renal failure: a pilot study. Nephrol Dial Transplant 27(5):1873-1880

Babbitt DM, Diaz KM, Feairheller DL, Sturgeon KM, Perkins AM, Veerabhadrappa P, Lee H (2013) Endothelial activation microparticles and inflammation status improve with exercise training in African Americans. Int J Hypertens 2013:1-8

Bakeman R (2005) Recommended effect size statistics for repeated measures designs. Behav Res Methods 37(3):379-384

Berg AH, Scherer PE (2005) Adipose tissue, inflammation, and cardiovascular disease. Circ Res 96(9):939-949

Burton JO, Hamali HA, Singh R, Abbasian N, Parsons R, Patel AK, Brunskill NJ (2013) Elevated levels of procoagulant plasma microvesicles in dialysis patients. PLoS One 8(8):e72663

Cortvrindt C, Speeckaert R, Moerman A, Delanghe JR, Speeckaert MM (2017) The role of interleukin-17A in the pathogenesis of kidney diseases. Pathology 49(3):247-258

Daniel L, Fakhouri F, Joly D, Mouthon L, Nusbaum P, Grunfeld J-P, Halbwachs-Mecarelli L (2006) Increase of circulating neutrophil and platelet microparticles during acute vasculitis and hemodialysis. Kidney Int 69(8):1416-1423

Daniel L, Dou L, Berland Y, Lesavre P, Mecarelli-Halbwachs L, Dignat-George $\mathrm{F}$ (2008) Circulating microparticles in renal diseases. Nephrol Dial Transplant 23(7):2129-2132

Delgado C, Johansen KL (2011) Barriers to exercise participation among dialysis patients. Nephrol Dial Transplant 27(3):1152-1157

Dungey M, Bishop NC, Young HM, Burton JO, Smith AC (2015) The impact of exercising during haemodialysis on blood pressure, markers of cardiac injury and systemic inflammationpreliminary results of a pilot study. Kidney Blood Press Res 40(6):593-604

Dungey M, Young HM, Churchward DR, Burton JO, Smith AC, Bishop NC (2017) Regular exercise during haemodialysis promotes an anti-inflammatory leucocyte profile. Clin Kidney J 10(6):813-821

Gleeson M, Bishop NC, Stensel DJ, Lindley MR, Mastana SS, Nimmo MA (2011) The anti-inflammatory effects of exercise: mechanisms and implications for the prevention and treatment of disease. Nat Rev Immunol 11(9):607-615

Gołębiowski T, Kusztal M, Weyde W, Dziubek W, Woźniewski M, Madziarska K, Klinger M (2012) A program of physical rehabilitation during hemodialysis sessions improves the fitness of dialysis patients. Kidney Blood Press Res 35(4):290-296 
Graham-Brown M, March D, Churchward D, Young H, Dungey M, Lloyd S, Burton JO (2016) Design and methods of CYCLE-HD: improving cardiovascular health in patients with end stage renal disease using a structured programme of exercise: a randomised control trial. BMC Nephrol 17(1):69

Graham-Brown MP, March DS, Young R, Highton PJ, Young HM, Churchward DR, Brunskill NJ (2021) A randomized controlled trial to investigate the effects of intra-dialytic cycling on left ventricular mass. Kidney Int. https://doi.org/10.1016/j.kint.2021.02. 027

Hashmi S, Zeng QT (2006) Role of interleukin-17 and interleukin17-induced cytokines interleukin-6 and interleukin-8 in unstable coronary artery disease. Coron Artery Dis 17(8):699-706

Hauser AB, Stinghen AE, Kato S, Bucharles S, Aita C, Yuzawa Y, Pecoits-Filho R (2008) Characteristics and causes of immune dysfunction related to uremia and dialysis. Perit Dial Int 28(Supplement 3):S183-S187

Heine GH, Ortiz A, Massy ZA, Lindholm B, Wiecek A, MartínezCastelao A, London GM (2012) Monocyte subpopulations and cardiovascular risk in chronic kidney disease. Nat Rev Nephrol $8(6): 362$

Highton PJ, Martin N, Smith AC, Burton JO, Bishop NC (2018) Microparticles and exercise in clinical populations. Exerc Immunol Rev 24:46-58

Highton PJ, Goltz FR, Martin N, Stensel DJ, Thackray AE, Bishop NC (2019) Microparticle responses to aerobic exercise and meal consumption in healthy men. Med Sci Sports Exerc 51(9):1935-1943

Highton PJ, White AE, Nixon DG, Wilkinson TJ, Neale J, Martin N, Smith AC (2020) Influence of acute moderate-to high-intensity aerobic exercise on markers of immune function and microparticles in renal transplant recipients. Am J Physiol Renal Physiol 318(1):F76-F85

Hoyer KK, Dooms H, Barron L, Abbas AK (2008) Interleukin-2 in the development and control of inflammatory disease. Immunol Rev 226(1):19-28

Hu ZB, Chen Y, Gong YX, Gao M, Zhang Y, Wang GH, Ma KL (2016) Activation of the CXCL16/CXCR6 pathway by inflammation contributes to atherosclerosis in patients with end-stage renal disease. Int J Med Sci 13(11):858

Kusano KF, Nakamura K, Kusano H, Nishii N, Banba K, Ikeda T, Miura A (2004) Significance of the level of monocyte chemoattractant protein-1 in human atherosclerosis. Circ J 68(7):671-676

Lannigan J, Erdbruegger U (2017) Imaging flow cytometry for the characterization of extracellular vesicles. Methods 112:55-67

Martin N, Smith AC, Dungey MR, Young HM, Burton JO, Bishop NC (2018) Exercise during hemodialysis does not affect the phenotype or prothrombotic nature of microparticles but alters their proinflammatory function. Physiol Rep 6(19):e13825

McIntyre CW, Burton JO, Selby NM, Leccisotti L, Korsheed S, Baker CS, Camici PG (2008) Hemodialysis-induced cardiac dysfunction is associated with an acute reduction in global and segmental myocardial blood flow. Clin J Am Soc Nephrol 3(1):19-26

Moser B, Roth G, Brunner M, Lilaj T, Deicher R, Wolner E, Ankersmit HJ (2003) Aberrant T cell activation and heightened apoptotic turnover in end-stage renal failure patients: a comparative evaluation between non-dialysis, haemodialysis, and peritoneal dialysis. Biochem Biophys Res Commun 308(3):581-585

Papayianni A, Alexopoulos E, Giamalis P, Gionanlis L, Belechri AM, Koukoudis P, Memmos D (2002) Circulating levels of ICAM-1,
VCAM-1, and MCP-1 are increased in haemodialysis patients: association with inflammation, dyslipidaemia, and vascular events. Nephrol Dial Transplant 17(3):435-441

Peres A, Perotto DL, Dorneles GP, Fuhro MIS, Monteiro MB (2015) Effects of intradialytic exercise on systemic cytokine in patients with chronic kidney disease. Ren Fail 37(9):1430-1434

Piccin A, Murphy WG, Smith OP (2007) Circulating microparticles: pathophysiology and clinical implications. Blood Rev 21(3):157-171

Rhind SG, Shek PN, Shinkai S, Shephard RJ (1996) Effects of moderate endurance exercise and training on in vitro lymphocyte proliferation, interleukin-2 (IL-2) production, and IL-2 receptor expression. Eur J Appl Physiol 74(4):348-360

Rysz J, Banach M, Cialkowska-Rysz A, Stolarek R, Barylski M, Drozdz J, Okonski P (2006) Blood serum levels of IL-2, IL-6, IL-8, TNF-alpha and IL-1beta in patients on maintenance hemodialysis. Cell Mol Immunol 3(2):151-154

Stenvinkel P, Ketteler M, Johnson RJ, Lindholm B, Pecoits-Filho R, Riella M, Girndt M (2005) IL-10, IL-6, and TNF- $\alpha$ : central factors in the altered cytokine network of uremia - the good, the bad, and the ugly. Kidney Int 67(4):1216-1233

Strasser EF, Happ S, Weiss DR, Pfeiffer A, Zimmermann R, Eckstein R (2013) Microparticle detection in platelet products by three different methods. Transfusion 53(1):156-166

Szatanek R, Baj-Krzyworzeka M, Zimoch J, Lekka M, Siedlar M, Baran J (2017) The methods of choice for extracellular vesicles (EVs) characterization. Int J Mol Sci 18(6):1153

Toussaint ND, Polkinghorne KR, Kerr PG (2008) Impact of intradialytic exercise on arterial compliance and B-type natriuretic peptide levels in hemodialysis patients. Hemodial Int 12(2):254-263

Trøseid M, Lappegård KT, Claudi T, Damås JK, Mørkrid L, Brendberg R, Mollnes TE (2004) Exercise reduces plasma levels of the chemokines MCP-1 and IL-8 in subjects with the metabolic syndrome. Eur Heart J 25(4):349-355

UK Renal Registry (2019) UK Renal Registry 21st Annual Report. Retrieved from Renal Association. ISSN 2632-4326. https://www. renalreg.org/patient-info/

Van der Pol E, Coumans F, Grootemaat A, Gardiner C, Sargent I, Harrison P, Nieuwland R (2014) Particle size distribution of exosomes and microvesicles determined by transmission electron microscopy, flow cytometry, nanoparticle tracking analysis, and resistive pulse sensing. J Thromb Haemost 12(7):1182-1192

Wong J, Davis P, Patidar A, Zhang Y, Vilar E, Finkelman M, Farrington $K$ (2017) The effect of intra-dialytic exercise on inflammation and blood endotoxin levels. Blood Purif 44(1):51-59

Zhang J, Hua G, Zhang X, Tong R, Du X, Li Z (2010) Regulatory T cells/T-helper cell 17 functional imbalance in uraemic patients on maintenance haemodialysis: a pivotal link between microinflammation and adverse cardiovascular events. Nephrology 15(1):33-41

Publisher's Note Springer Nature remains neutral with regard to jurisdictional claims in published maps and institutional affiliations. 